\title{
SOME REMARKS ON POSITIVE VECTOR BUNDLES
}

\author{
ROBERT FRANKEL
}

Let $E$ be a holomorphic vector bundle over a compact complex manifold. If $E$ admits a hermitian metric whose curvature satisfies a certain analytic condition which we describe in $\$ 1$ below, then $E$ is said to be positive. In this note, we prove two results on positive vector bundles.

Positive vector bundles were investigated by Griffiths in [3]. He conjectured that a bundle is positive if and only if it is ample in the sense of Hartshorne. Although Griffiths showed that positive bundles are ample, and although his conjecture has been proven for bundles over Riemann surfaces (see [7]), the conjecture in general remains unsolved. To some extent, the intractability of this problem can be traced to the fact that we do not know many "functorial" properties of positive bundles, such as the properties that Hartshorne proves for ample bundles in [4]. In \$1, we establish such a functorial property: We show that if one pulls a positive bundle back under a finite mapping, one obtains a positive bundle (Theorem 1.1). Hartshorne proved the analogue for ample bundles [4, p. 73, Proposition 4.3].

Another functorial result Hartshorne proved for ample bundles is

Theorem A [4, p. 73, Proposition 4.4]. Let $f: X \rightarrow Y$ be a proper morphism, and let $E$ be a bundle on $X$. Suppose that for some $y \in Y$, the restriction of $E$ to the fiber $f^{-1}(y)$ is ample. Then there exists a neighborhood $U$ of $y$ such that the restriction of $E$ to $f^{-1}(U)$ is ample.

This theorem immediately yields the weaker

Theorem B. Given the notation and assumptions of Theorem A, let $K$ be a bundle on $X$. Then there is a neighborhood $U$ of $y$ such that if $y^{\prime} \in U$, then

$$
H^{q}\left(f^{-1}\left(y^{\prime}\right), E^{(\mu)} \otimes K\right)=0 \text { for all } q>0 \text { and } \mu \geqslant \mu\left(y^{\prime}\right),
$$

$\mu\left(y^{\prime}\right)$ being a positive integer depending on $y^{\prime}$.

In §2, under the (possibly) stronger assumption that the restriction of $E$ to $f^{-1}(y)$ is positive, together with an assumption on the rank of $f$, we show that one can choose $\mu\left(y^{\prime}\right)$ to be the same integer for all $y^{\prime} \in U$ (Theorem 2.1).

Received February 7, 1977. 
The two sections can be read independently, except that the first paragraph of $\S 1$, in which the basic definitions are given, should be read before $\S 2$.

The author thanks David Lieberman and Peter Orlik for their invaluable advice and encouragement.

\section{Behavior of positive bundles under finite mappings}

Let $E$ be a holomorphic vector bundle over a compact complex manifold, and let $h$ be a hermitian metric on $E$. Let $\theta$ denote the curvature of $h$; that is, $\theta$ is the $\operatorname{Hom}(E, E)$-valued $(1,1)$-form which is given locally by $\theta=\bar{\partial}\left(h^{-1} \partial h\right)$. We say that $h$ is positively curved if for any $e \in E, e \neq 0, h(\theta e, e)$ is a positive $(1,1)$-form. (A $(1,1)$-form $\xi$ is positive if for any nonzero holomorphic tangent vector $v$, we have $\xi(v \wedge \vec{v})>0$.) If the bundle $E$ admits a metric $h$ which is positively curved, $E$ is said to be positive.

Theorem 1.1. Let $f: X \rightarrow Y$ be a finite mapping of compact complex manifolds, and let $E$ be a holomorphic vector bundle over $Y$. Then $E$ positive implies $f^{*} E$ positive.

The proof involves the following simple lemma.

Lemma 1.2. Let $E$ be a holomorphic vector bundle over a complex manifold $X$. Suppose $E$ admits a metric $h$ which is positively curved except at a point $z_{0} \in X$. Then there exists a $C^{\infty}$ function $g: X \rightarrow \mathbf{R}$ such that the metric $e^{8} h$ is positively curved everywhere, and such that $e^{g} h=h$ outside of a presassigned neighborhood of $z_{0}$.

Proof of the lemma. The result is local in nature, so we may assume $X=\mathbf{C}^{n}, E=$ the trivial bundle $\theta^{r}$, and $z_{0}=0$. We now construct the function $g$ required in the statement of the lemma.

Let $\rho: \mathbf{R} \rightarrow \mathbf{R}$ be a compactly supported $C^{\infty}$ function satisfying: (i) $\rho(0)>$ 0 , (ii) $\rho^{\prime}(0)=0$, and (iii) $\rho^{\prime \prime}(0)<0$. In $\mathbf{C}^{n}$, let $z_{1}, \cdots, z_{n}$ be coordinates with $z_{j}=x_{j}+\sqrt{-1} y_{j}$. Define

$$
\zeta(z)=\rho\left(x_{1}\right) \rho\left(x_{2}\right) \cdots \rho\left(x_{n}\right) \rho\left(y_{1}\right) \cdots \rho\left(y_{n}\right) .
$$

Then $\zeta$ has compact support, and a direct computation shows that the matrix $\left\|\partial^{2} \zeta / \partial z_{i} \partial \bar{z}_{j}\right\|_{i, j}$ is negative-definite at $z=0$. So for some $R>0$, $\partial \partial \zeta$ is a positive $(1,1)$-form for $|z|<R$.

Let $\varepsilon>0$, and consider the metric $e^{e s} h$ on $\vartheta^{r}$. Its curvature is (locally) $\theta(h)+(\varepsilon \bar{\partial} \partial \zeta) I$, where $I$ is the $r \times r$ identity matrix. So for sufficiently small $\varepsilon$, this curvature is positive when $|z| \geqslant R / 2$. But for $|z|<R, \theta(h)$ is semipositive and $\varepsilon \bar{\partial} \partial \zeta I$ is positive. Thus for $\varepsilon$ small, $e^{\varepsilon \zeta h}$ has positive curvature everywhere, and we let $g=\varepsilon \zeta$.

Proof of the theorem. We are given a positive bundle $E$, and want to show 
that its pullback under a finite mapping $f$ is positive. Let $h^{\prime}$ be a metric on $E$ with positive curvature, and let $h=f^{*} h^{\prime}$, a metric on $f^{*} E$. We are going to show that $e^{\varepsilon Z} h$ is positively curved, for some function $Z$ on $X$ and $\varepsilon>0$. Let us begin by seeing how close $h$ itself comes to being positively curved. We compute the curvature of $h$ : Let $x \in X, e \in\left(f^{*} E\right)_{x}$, and let $v$ be a holomorphic tangent vector at $x$ with $v \neq 0$ and $e \neq 0$. If $h$ were positively curved, we would have

$$
h(\theta(h) e, e)(v \wedge \bar{v})>0 .
$$

As it is, using the fact that $\theta(h)=f^{*} \theta\left(h^{\prime}\right)$ we compute that $h(\theta(h) e, e)(v \wedge v)$ $>0$ if and only if $v \notin K=\operatorname{ker} f_{*}, f_{*}: T X \rightarrow T Y$ being the induced mapping on holomorphic tangent bundles. (In particular, if $D$ is the ramification divisor of $f$, then $h$ is positively curved away from $D$.) Therefore one should intuitively think of the situation in the following way: Locally, imagine a tubular neighborhood of $D$. On any transverse fiber of this neighborhood, $h$ is positively curved, except at the point where the fiber meets $D$. This suggests that $Z$ be constructed by patching together functions which, in normal directions to $D$, behave like the function $\zeta$ we constructed in the proof of the lemma.

We now proceed to construct $Z$. Let $\left\{V_{\alpha}\right\}$ be a covering of $X$ by coordinate patches such that for each $\alpha, V_{\alpha} \subset \subset U_{\alpha}$, where $U_{\alpha} \subset X$ is also a coordinate patch. On $U_{\alpha}$, perform the construction of the function $\zeta$ in the proof of the lemma. Call this function $\zeta_{\alpha}$, and arrange it that $\bar{\partial} \partial \zeta_{\alpha}$ is a positive $(1,1)$-form on $V_{\alpha}$. Now let $\left\{\rho_{\alpha}^{\prime}\right\}$ be a partition of unity on $Y$ subordinate to the cover $\left\{f\left(V_{\alpha}\right)\right\}$, and set $\rho_{\alpha}=f^{*} \rho_{\alpha}^{\prime}$. Finally, let $Z=\Sigma \rho_{\alpha} \zeta_{\alpha}$.

We have

$$
\theta\left(e^{\varepsilon Z} h\right)=\theta(h)+(\varepsilon \bar{\partial} \partial Z) I,
$$

$I$ being the $r \times r$ identity matrix. $\bar{\partial} \partial Z$ is made up of terms of four types: (a) $\left(\bar{\partial} \partial \rho_{\alpha}\right) \zeta_{\alpha}$, (b) $\bar{\partial} \rho_{\alpha} \partial \zeta_{\alpha}$, (c) $\partial \rho_{\alpha} \bar{\partial} \zeta_{\alpha}$, and (d) $\rho_{\alpha} \bar{\partial} \partial \zeta_{\alpha}$. If $v \in K=\operatorname{ker} f_{*}$, then any of the types (a), (b), and (c) give zero when evaluated on $v \wedge \bar{v}$. For, $d \rho_{\alpha}(v)=$ $d \rho_{\alpha}^{\prime}\left(f_{*} v\right)=0$ since $f_{*} v=0$. But the nonvanishing term $\Sigma \rho_{\alpha} \bar{\partial} \partial \zeta_{\alpha}$ will be positive on $K$, since $\rho_{\alpha}=0$ off of $V_{\alpha}$ and $\bar{\partial} \partial \zeta_{\alpha}$ is positive on $V_{\alpha}$.

So for any $x \in D, \varepsilon \bar{\partial} \partial Z$ is positive on $K_{x}$, and (by continuity) is in fact positive on a cone in $T_{x} X$ containing the subspace $K_{x}$; and this cone will not vary with $\varepsilon$. Therefore, for any $\varepsilon>0, \theta\left(e^{\varepsilon Z} h\right)$ will be positive on this cone. Choose $\varepsilon$ so small that $\theta\left(e^{\varepsilon Z} h\right)$ will also be positive on the complement, in $T_{x} X$, of a slightly smaller cone. This can be done since $\theta(h)$ itself is positive on this complement. (By compactness, a single $\varepsilon$ will work for all $x \in D$.) For such $\varepsilon$, we therefore see that $\theta\left(e^{\varepsilon Z} h\right)$ is positive on $T X$ restricted to $D$, and (again by continuity) in fact on $T X$ restricted to $N$, where $N$ is some 
neighborhood of $D$. It is clear that $N$ will not shrink as $\varepsilon \rightarrow 0$. We may think of $N$ as the neighborhood of radius $R$ for some $R>0$, with respect to some metric $d$ on $X$. That is,

$$
N=\{x \in X: d(x, D)<R\} .
$$

Choose $\varepsilon$ so small that $\theta\left(e^{\varepsilon Z} h\right)$ is positive on the complement (in $X$ ) of the neighborhood of $D$ of radius $R / 2$, and the proof is complete.

Corollary 1.3. Let $f: X \rightarrow Y$ be a finite mapping of compact complex manifolds. If $Y$ is Kahler, so is $X$.

This corollary is in fact well known. The usual proof follows from a theorem of Blanchard [5, p. 142]. Our proof is more elementary.

Proof of the corollary. Let $\omega^{\prime}$ be a Kahler form on $Y$, and let $\omega=f^{*} \omega^{\prime}$. In the proof of the theorem, we defined a function $Z$ and proved that $\theta(h)+$ $(\varepsilon \bar{\partial} \partial Z) I$ was a positive form for sufficiently small $\varepsilon$. The same proof shows that $\omega+\varepsilon \bar{\partial} \partial Z$ is a positive $(1,1)$-form on $X$, with the same $Z$ as in the proof of the theorem. Since $d(\omega+\varepsilon \bar{\partial} \partial Z)=0, \omega+\varepsilon \bar{\partial} \partial Z$ is a Kahler form on $X$.

\section{A vanishing theorem}

The theorem we are headed for is

Theorem 2.1. Let $f: X \rightarrow Y$ be an analytic family of complex manifolds. (That is, $f$ is proper, and of maximal rank at every point of $X$.) Let $E$ be a holomorphic vector bundle over $X$, and suppose that for some $y \in Y$ we have that $E(y)$ is positive, where $E(y)$ denotes the restriction of $E$ to $f^{-1}(y)$. If $K$ is a holomorphic vector bundle over $X$, then there exists a neighborhood $U$ of $y$ and $\mu_{0}>0$ such that

$$
H^{q}\left(f^{-1}\left(y^{\prime}\right), E^{(\mu)} \otimes K\right)=0 \text { for } q>0, \mu \geqslant \mu_{0}, \text { and } y^{\prime} \in U,
$$

where $E^{(\mu)}$ denotes the $\mu$ th symmetric power of $E$.

We begin by proving the theorem for the special case where $E$ is a line bundle. The proof is really an elaboration of an argument of Griffiths [3, pp. 212-213], although the theorem he was proving is of a nature somewhat different from our Theorem 2.1.

By Kodaira-Serre duality, it suffices to prove

$$
H^{q}\left(f^{-1}\left(y^{\prime}\right), E^{-\mu} \otimes K\right)=0 \text { for } q<n, \mu \geqslant \mu_{0} \text {, and } y^{\prime} \in U .
$$

Let $h_{E}$ be a positively curved hermitian metric on $E(y)$. Since $h_{E}$ is a $C^{\infty}$ structure, it can be extended to all of $E$. (This is the only place we use the assumption that $f$ has maximal rank everywhere. The maximality of rank implies that, in terms of $C^{\infty}$ structure, $f$ exhibits $X$ as a local product over $Y$.) By continuity and compactness, $h_{E}$ is positive on $E\left(y^{\prime}\right)$ for all $y^{\prime}$ in some neighborhood $V$ of $y$.

Now let $h_{K}$ be a hermitian metric on $K$. For any $y^{\prime} \in Y$, let $\theta_{K}\left(y^{\prime}\right)$ and 
$\theta_{E}\left(y^{\prime}\right)$ denote the curvatures, with respect to $h_{K}$ and $h_{E}$, of $K\left(y^{\prime}\right)$ and $E\left(y^{\prime}\right)$ respectively. Given any $y^{\prime} \in V, h_{E}\left(y^{\prime}\right)$ and $h_{K}\left(y^{\prime}\right)$ determine a Hodge inner product on $E\left(y^{\prime}\right)^{-\mu} \otimes K\left(y^{\prime}\right)$-valued forms. Let $\Lambda\left(y^{\prime}\right)$ denote the dual of the operator $\theta_{E}\left(y^{\prime}\right)$ with respect to this inner product. Let

$$
\Psi\left(y^{\prime}\right)=(\sqrt{-1} / 2) \Lambda\left(y^{\prime}\right) \theta_{K}\left(y^{\prime}\right) .
$$

Then $\Psi\left(y^{\prime}\right)$ is an operator on $E\left(y^{\prime}\right)^{-\mu} \otimes K\left(y^{\prime}\right)$-valued forms, bounded with respect to the Hodge inner product. Denoting the norm of this operator by $\left|\Psi\left(y^{\prime}\right)\right|$, one obtains

$$
\left(\Psi\left(y^{\prime}\right) \wedge \phi, \phi\right) \geqslant \mu(n-q)(\phi, \phi),
$$

where $\phi$ is an $E\left(y^{\prime}\right)^{-\mu} \otimes K\left(y^{\prime}\right)$-valued $(0, q)$-form, and (, ) is the Hodge inner product. (See [3, p. 213, line 3.30].) Hence if $\phi \neq 0$,

$$
\left|\Psi\left(y^{\prime}\right)\right| \geqslant \mu(n-q) \geqslant \mu \text {. }
$$

Therefore if $\mu_{0}>\left|\Psi\left(y^{\prime}\right)\right|$, then

$$
H^{q}\left(f^{-1}\left(y^{\prime}\right), E^{-\mu} \otimes K\right)=0 \text { for } q<n \text { and } \mu \geqslant \mu_{0} .
$$

So, fix any $\mu_{0}>|\Psi(y)|$. Then by continuity, $\mu_{0}>\left|\Psi\left(y^{\prime}\right)\right|$ for any $y^{\prime}$ in some neighborhood $U$ of $y, U \subset V$. This completes the proof of the line bundle version of Theorem 2.1.

To prove Theorem 2.1 in the general case, we need a lemma.

Lemma 2.2. Let $E$ be a holomorphic vector bundle over the compact complex manifold $X$. Let $p: P(E) \rightarrow X$ denote the associated projective bundle of hyperplanes in $E$, and let $L=L(E)$ be the tautological (hyperplane) line bundle over $P(E)$. Let $K$ be a holomorphic vector bundle over $X$. Then

$$
H^{q}\left(X, E^{(\mu)} \otimes K\right)=H^{q}\left(P(E), L^{\mu} \otimes p^{*} K\right) \text { for all } q \text {, and } \mu \geqslant \mu_{0} \text {. }
$$

This lemma is standard: It essentially amounts to the statement that $E$ is ample if and only if $L(E)$ is. But we give a proof here which uses the line bundle version of Theorem 2.1, and which is more elementary than the usual proofs. For instance, Griffiths uses Kohn's theory of harmonic integrals to prove this result in [2, pp. 135-136].

Proof of the lemma. We have the Leray spectral sequence for $p$ with

$$
E_{2}^{q t} \stackrel{\bullet}{=} H^{q}\left(X, R_{p}^{t}\left(L^{\mu} \otimes p^{*} K\right)\right) \text { and } E_{\infty} \cong H^{*}\left(P(E), L^{\mu} \otimes p^{*} K\right) .
$$

If we can show

$$
R_{p}^{t}\left(L^{\mu} \otimes p^{*} K\right)=0 \text { for } t>0 \text { and } \mu \geqslant \mu_{0},
$$

then we will have, by the standard spectral sequence degeneration argument,

$$
H^{q}\left(X, p_{*}\left(L^{\mu} \otimes p^{*} K\right)\right)=H^{q}\left(P(E), L^{\mu} \otimes p^{*} K\right) .
$$

But the left side of this equation is $H^{q}\left(X, E^{(\mu)} \otimes K\right)$, so once we show (*) we will be done with the proof of the lemma. 
To prove (*), we first fix $x \in X$. Since the restriction of $L$ to $p^{-1}(x)$ is just the standard hyperplane bundle on projective space and is therefore ample, it follows from the definition of ampleness that

$$
H^{t}\left(p^{-1}(x), L^{\mu} \otimes p^{*} k\right)=0 \text { for } t>0 \text { and } \mu \geqslant \mu_{0} \text {. }
$$

Therefore we can conclude from the line bundle version of Theorem 2.1 that there exists a neighborhood $U$ of $x$ such that

$$
H^{t}\left(p^{-1}\left(x^{\prime}\right), L^{\mu} \otimes p^{*} K\right)=0 \text { for } t>0, \mu \geqslant \mu_{0} \text {, and } x^{\prime} \in U .
$$

Thus by compactness of $X$ we can actually conclude that there exists $\mu_{0}>0$ such that for all $x \in X$, we have

$$
H^{t}\left(p^{-1}(x), L^{\mu} \otimes p^{*} K\right)=0 \text { for } t>0 \text { and } \mu \geqslant \mu_{0} .
$$

We now obtain (*) by invoking [6, Corollary 2, p. 50$]$.

Remark. Although this result of Mumford is stated in the language of schemes, his proof goes through for complex manifolds by using Leray covers where he uses affine covers. Furthermore, we can give a proof of this lemma which avoids Mumford's result, but which uses the obstruction theory described in [1, pp. 369-372].

Proof of Theorem 2.1. Let $p: P(E) \rightarrow X$ denote the associated projective bundle, and let $L=L(E) . f p$ is an analytic family, so by the line bundle version of Theorem 2.1, we have

$$
H^{q}\left(p^{-1} f^{-1}\left(y^{\prime}\right), L^{\mu} \otimes p^{*} K\right)=0 \quad \text { for } q>0, \mu \geqslant \mu_{0}, \text { and } y^{\prime} \in U .
$$

But according to Lemma 2.2,

$$
H^{q}\left(f^{-1}\left(y^{\prime}\right), E^{(\mu)} \otimes K\right)=H^{q}\left(p^{-1} f^{-1}\left(y^{\prime}\right), L^{\mu} \otimes p^{*} K\right),
$$

so we are done.

\section{Bibliography}

[1] P. Griffiths, The extension problem in complex analysis. II, Amer. J. Math. 88 (1966) 366-446.

[2] Hermitian differential geometry and the theory of positive and ample holomorphic vector bundles, J. Math. Mech. 14 (1965) 117-140.

[3] _ Hermitian differential geometry, Chern classes, and positive vector bundles, Global Analysis, Princeton University Press, Princeton, 1969, 185-251.

[4] R. Hartshorne, Ample vector bundles, Inst. Hautes Etudes Sci. Publ. Math. No. 29 (1966) 319-350.

[5] F. Hirzebruch, Topological methods in algebraic geometry, 3rd ed., Springer, New York, 1966.

[6] D. Mumford, Albelian varieties, (Published for Tata Institute of Fundamental Research, Bombay) Oxford University Press, Oxford, 1970.

[7] H. Umemura, Some results in the theory of vector bundles, Nagoya Math. J. 52 (1973) 97-128.

UnIVERSITY OF MASSACHUSETTS, Boston 